\title{
Skin Test Reactivity to Indoor Allergens Correlates with Asthma Severity in Jeddah, Saudi Arabia
}

\author{
Emad A. Koshak, MD, FRCPSC
}

\begin{abstract}
Background: There is increased emphasis on the role of indoor allergens in asthma.

Objective: To examine the spectrum of skin test reactivity (sensitization) to indoor allergens and its correlation with asthma severity in Jeddah, Saudi Arabia.

Methods: Asthmatic patients referred to the allergy clinic at King Abdulaziz University Hospital (KAUH) in Jeddah were studied. Measures of clinical severity were adopted from national and international asthma guidelines. The degree of sensitization was assessed by the wheal size (positive $\geq 3 \mathrm{~mm}$ ) from standard skin-prick tests for the following common indoor inhalant allergens: house dust mites (Dermatophagoides pteronyssinus [Dp] and Dermatophagoides farinae [Df]), cat, and cockroach.

Results: Skin test results from 113 of $151(74.8 \%)$ asthmatic patients were positive for one or more allergens. The patients' ages ranged between 9 and 63 years (mean, $30 \pm 13$ years), and females constituted $65.5 \%$. The predominant asthma severity level was moderate persistent $(55.8 \%)$, followed by mild persistent (33.6\%). The prevalences of sensitization to indoor allergens were as follows: $D p, 87 \%$ (3-25 mm [mean, $7 \mathrm{~mm}]$ ); Df, 84\% (3-20 mm [mean, $7 \mathrm{~mm}]$ ); cat, 44\% (3-15 mm [mean, $6 \mathrm{~mm}]) ;$ and cockroach, 33\% (3-12 mm [mean, $4 \mathrm{~mm}])$. Higher asthma severity levels were significantly correlated with the number of allergens with positive sensitization $(R=0.3, p<.001)$ and with the degree of sensitization to house dust mites $(D p$ [degrees of freedom $\{d f\}=16, p<.001]$ and $D f[d f=17, p<.01])$ but not to cat $(d f=10, p<.24)$ or cockroach $(d f=8, p<.36)$.

Conclusions: Immunoglobulin E-mediated skin test reactivity to indoor allergens, particularly to house dust mites, was common in asthmatic patients from Jeddah at KAUH. Increased sensitization was associated with higher levels of asthma severity, which is compatible with the literature. This emphasizes the importance of identifying sensitization to relevant indoor allergens in the clinical evaluation of asthmatic persons.
\end{abstract}

E.A. Koshak-Associate Professor and Consultant in Internal Medicine, Allergy, Asthma, and Clinical Immunology, Department of Internal Medicine, Faculty of Medicine, King Abdulaziz University Hospital, Jeddah, Saudi Arabia

Correspondence to: Dr. Emad A. Koshak, Department of Internal Medicine, King Abdulaziz University Hospital, P.O. Box 80215, Jeddah, 21589, Kingdom of Saudi Arabia

DOI 10.2310/7480.2006.00002
Asthmatic airways are characterized by an immunologic chronic inflammation that has been documented to occur after exposure to an allergen. ${ }^{1,2}$ Several studies have suggested a correlation between allergen exposure and the prevalence of asthma. ${ }^{3-5}$ In sensitized individuals, exposure to airborne allergens is a risk factor for asthma exacerbations, the persistence of asthma symptoms, and significant changes in pulmonary function. ${ }^{6-8}$

Worldwide, the documented increase in the prevalence of asthma has been almost entirely an increase in that of perennial asthma, and a 
Table 1 Criteria Used for Categorizing the Clinical Severity of Asthmatic Patients

\begin{tabular}{llll}
\hline Severity Level & Day Symptoms & Night Symptoms & PEF \\
\hline Mild intermittent & $\leq 2$ times/week & $\leq 2$ times/month & $\geq 80 \%$ \\
Mild persistent & $>2$ times/week but $<1$ time/day & $>2$ times/month & $\geq 80 \%$ \\
Moderate persistent & Daily & $>1$ time/week & $>60$ to $<80 \%$ \\
Severe persistent & Continuous & Frequent & $\leq 60 \%$ \\
\hline
\end{tabular}

$\mathrm{PEF}=$ peak expiratory flow.

large proportion of such patients are allergic to allergens that are found all year round in homes. ${ }^{9,10}$ This has led to increased interest in the immunologic role of allergenic substances that accumulate indoors. Indoor allergens today have increased in developed countries where homes have been insulated for energy efficiency, carpeted, heated, cooled, and humidified—changes that have also made homes an ideal habitat for the generators of indoor allergens. ${ }^{11,12}$

Globally, the most abundant indoor allergens include those derived from house dust mites (HDMs), cats, and cockroaches. ${ }^{12,13}$ The principal HDM species are the pyroglyphid mites (Dermatophagoides pteronyssinus $[D p]$, Dermatophagoides farinae [Df], and others), which usually account for $90 \%$ of mite species in house dust in temperate regions. ${ }^{9,14}$ According to some local studies, similar allergens have been isolated from the homes of asthmatic persons living in different regions of Saudi Arabia. ${ }^{15}$

Recent progress in the fields of allergy and immunology have promoted extensive studies on the identification of sensitization to indoor allergens. In vivo and in vitro allergy tests are considered valuable tools for exploring the presence of an immunoglobulin E (IgE)-mediated immune response in atopic disorders such as bronchial asthma, and their results are a reflection of sensitization to the given allergen. ${ }^{16-18}$ Previous investigators demonstrated a direct relationship between positive allergy skin test reactions or sensitization to inhalant indoor allergens and the increase in the severity of asthma. ${ }^{19,20}$ However, no study has focused primarily on the clinical severity of asthma (assessed by international and national asthma management guidelines) in relation to common indoor allergens detected in Saudi Arabia.

Hence, this study aimed to explore the spectrum of IgE-mediated skin test reactivity or sensitization to common indoor inhalant allergens among asthmatic patients seen at King Abdulaziz University Hospital (KAUH) in Jeddah, the second-largest city in Saudi Arabia. This work focuses on the hypothesis that skin test reactivity to indoor allergens may have a relationship with higher levels of clinical severity of asthma.

\section{Methods}

This was a cross-sectional study on 151 patients with a primary diagnosis of bronchial asthma. They were sequentially selected from patients examined at the allergy clinic of KAUH from January 1997 to December 1999. Only patients who were living in the city of Jeddah were included in the study. Jeddah is a coastal city located in the western region of the Kingdom of Saudi Arabia on the Red Sea, and high humidity characterizes its weather, particularly in the summer season. The criteria for the diagnosis and assessment of the clinical severity of each asthmatic patient were daytime symptoms, nocturnal symptoms, and mean peak expiratory flow at the clinic visit (Table 1). These criteria were adopted from the National Asthma Education Prevention Program in the United States and the Saudi national protocol for the management of asthma. ${ }^{21,22}$ The presence of one of the features of severity is sufficient to place a patient in that severity category. Each asthmatic patient was assigned to the most severe grade in which any of their features occurred. 
Table 2 Frequency of Asthma Clinical Severity Levels

\begin{tabular}{lccc}
\hline Severity Level & No. of Patients & \% of Patients & Cumulative \% \\
\hline Mild intermittent & 8 & 7.1 & 7.1 \\
Mild persistent & 38 & 33.6 & 40.7 \\
Moderate persistent & 63 & 55.8 & 96.5 \\
Severe persistent & 4 & 3.5 & 100.0 \\
$\quad$ Total & 113 & 100.0 & - \\
\hline
\end{tabular}

Demographic data, documented asthma severity level assessments, and the results of skin-prick tests (SPTs) for common inhalant allergens were extracted from the records of the patients. The assessment of the level of asthma severity was carried out and documented in each patient's chart before the SPT was performed. The following patients were excluded from the study:

- Smokers (to rule out chronic obstructive airway disease)

- Patients who were pregnant or taking $\beta$-blockers (for safety reasons)

- Patients taking antihistamines or taking long-term oral corticosteroids for more than 1 week (because of their negative effect on SPT results)

Skin test reactivity (sensitization) was determined by reviewing the results of reactions to a standard SPT after 15 minutes. Verbal consent was obtained from candidates prior to skin-prick testing. The panel of indoor allergen extracts appropriate for the SPTs was obtained from Greer Laboratories in the United States. The indoor allergens used were the two common species of HDM ( $D p$ and $D f)$ and cat and cockroach extracts. The cockroach extract contained a 1:10 (weight per volume) mixture of two common species, Blattella germanica (German cockroach) and Periplaneta americana (American cockroach). Extracts of the HDMs ( $D p$ and $D f)$ and cat hair exist in concentrations of 10,000 allergen units per millilitre.

After sterilization of the forearm with propyl alcohol, single drops of each allergen extract were applied, $2 \mathrm{~cm}$ apart. A skin-prick test was performed within the allergen drops on the skin with a 26-gauge needle. Additionally, a drop of histamine phosphate (at a concentration of $2.7 \mathrm{mg} / \mathrm{mL}$ ) and a drop of the diluent were used as a positive control and a negative control, respectively. A wheal $\geq 5 \mathrm{~mm}$ in size in reaction to histamine was considered adequate for the competency of the test. A wheal $\geq 3 \mathrm{~mm}$ in diameter (more than the negative control) was considered a positive test result for sensitization to that peculiar allergen. The size of wheal for each indoor allergen was recorded and was used as a reflection of the degree of skin test reactivity (sensitization).

The degree of skin test reactivity for each case was classified, according to the diameter of its wheal, as mild sensitization $(3-5 \mathrm{~mm})$, moderate sensitization $(6-10 \mathrm{~mm})$, or severe sensitization $(\geq 11 \mathrm{~mm})$. Furthermore, the number of indoor allergens yielding a positive reaction was recorded for each case.

The data were entered into a personal computer. Frequency tables, correlation analysis by Pearson's test, and analysis of variance were performed with SPSS version 11 statistical software.

\section{Results}

Of 151 asthmatic individuals living in Jeddah, 113 had positive skin test reactions to one or more indoor allergens, accounting for $74.8 \%$ of the studied group. The ages of the patients who had positive skin test results ranged between 9 and 63 years (mean, $30 \pm 13$ standard deviations [SD]), and $74(65.5 \%)$ were female. The predominant levels of asthma severity were moderate persistent (63 cases [55.8\%]) and mild persistent (38 cases [33.6\%]) (Table 2). 
Table 3 Prevalence of Positive Skin Reactivity to the Different Indoor Allergens

\begin{tabular}{lcccc}
\hline Allergen & No. of Patients & \% of Patients & Wheal Diameter $(\mathrm{mm})$ & Mean \pm SD \\
\hline HDM $(D p)$ & 98 & 87 & $3-25$ & $7 \pm 4$ \\
HDM $(D f)$ & 95 & 84 & $3-20$ & $7 \pm 4$ \\
Cat & 50 & 44 & $3-15$ & $6 \pm 2$ \\
Cockroach & 38 & 33 & $3-12$ & $4 \pm 2$ \\
\hline
\end{tabular}

$D f=$ Dermatophagoides farinae $; \mathrm{D}=$ Dermatophagoides pteronyssinus; $\mathrm{HDM}=$ house dust mite.

The prevalence of positive skin reactivity and wheal size was highest for both HDMs: 98 cases (87\%) for $D p$ and 95 cases (84\%) for $D f$ (Table 3). Sensitization to more than one allergen, and particularly to two and three different allergens, was common (Table 4).

The frequency of asthma cases in each category of sensitization degree is shown in Figure 1. There was a correlation between sensitization to the two species of HDM ( $D f$ and $D p, p<.001)$, which represents a known cross-reactivity between them.

Statistically, the increasing levels of clinical severity in the asthmatic patients under study were significantly correlated to the number of indoor allergens yielding positive skin test reactivity (sensitization) $(\mathrm{R}=0.3, p<.001)$ and to the degree of skin test reactivity (ie, wheal size) to $D p(d f=16$, $p<.001)$ and $D f(d f=17, p<.01)$ but did not reach statistical significance in the cases of cat $(d f=10$, $p<.24)$ and cockroach $(d f=8, p<.36)$ allergens.

The relation between the severity of asthma and the degree of skin test reactivity to the different indoor allergens is shown in Figure 2. The linear relation between the severity of asthma and the mean degree of skin test reactivity is illustrated in Figure 3. Figure 4 shows that higher levels of asthma severity were associated with higher numbers of sensitization to indoor allergens.

\section{Discussion}

Our predominantly sedentary indoor lifestyle has been identified as one of the probable causes for increases in the prevalence of asthma. This indoor lifestyle may have led to either an increased exposure to allergens or an increase in factors that enhance the lungs' response to foreign proteins. ${ }^{9}$ Clearly, exposure to allergens can provoke acute asthma attacks as well as chronic allergic symptoms. ${ }^{10}$

In this study, positive skin test reactions to common indoor allergens were seen in up to threequarters of the enrolled asthmatic patients who were referred to the allergy clinic. Although such a high rate of sensitization is compatible with some international figures, selection bias must be considered. ${ }^{9,23}$ The majority of patients seen at the allergy clinic have moderate persistent asthma whereas most asthmatic persons in the general population have mild asthma. Doctors refer patients whom they suspect to have allergies to the allergy clinic, or patients request allergy assessment if they suspect themselves to have an allergy. In

Table 4 Number of Allergens Yielding Positive Skin Test Reactivity (Sensitization)

\begin{tabular}{lccc}
\hline & No. of Patients & \% of Patients & Cumulative \% \\
\hline Sensitization to 1 allergen & 17 & 15.0 & 15.0 \\
Sensitization to 2 allergens & 39 & 34.5 & 49.6 \\
Sensitization to 3 allergens & 40 & 35.4 & 85.0 \\
Sensitization to 4 allergens & 17 & 15.0 & 100.0 \\
Total & 113 & 100.0 & - \\
\hline
\end{tabular}



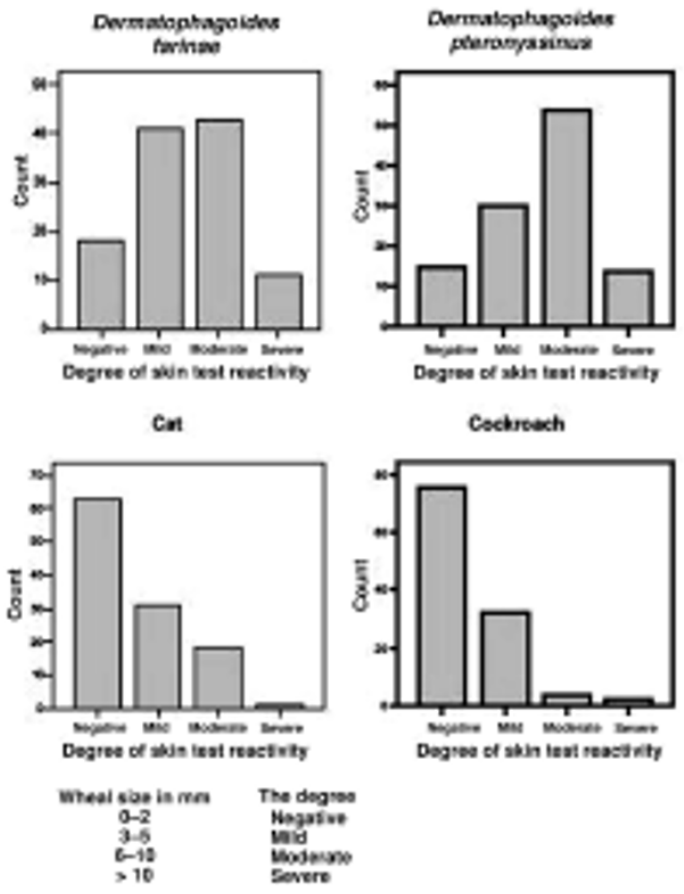

Figure 1 The degree of skin test reactivity (sensitization) to the indoor allergens studied. Wheal size was used as the measure of the degree of skin test reactivity (0-2 mm, negative; $3-5 \mathrm{~mm}$, mild; 6-10 mm, moderate; $>10 \mathrm{~mm}$, severe).
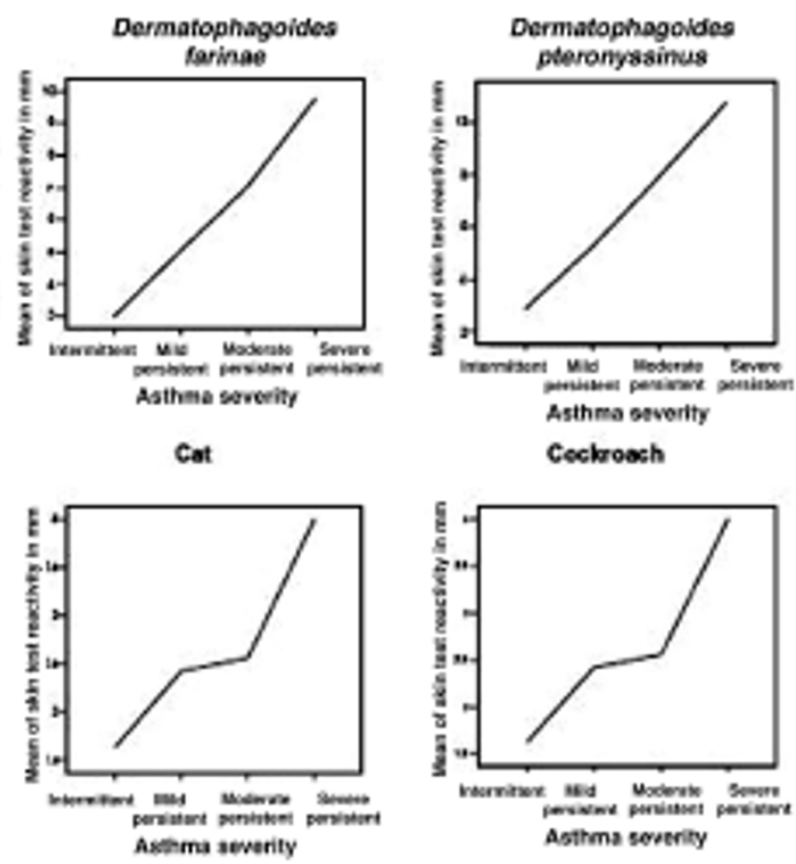

Figure 3 Mean skin test reactivity (wheal size in millimetres) to indoor allergens studied.

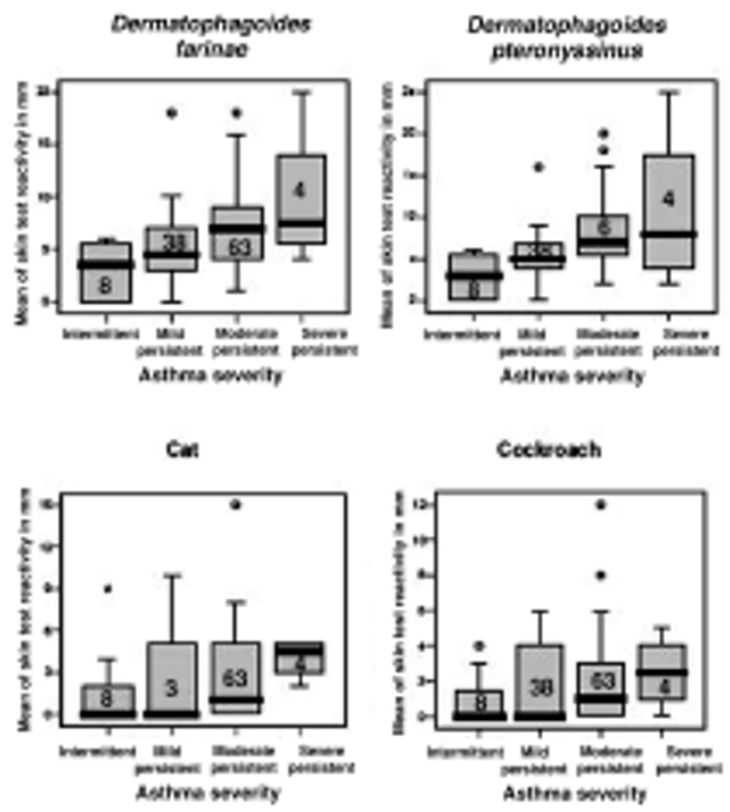

Figure 2 Box plot of skin test reactivity to the different indoor allergens studied.

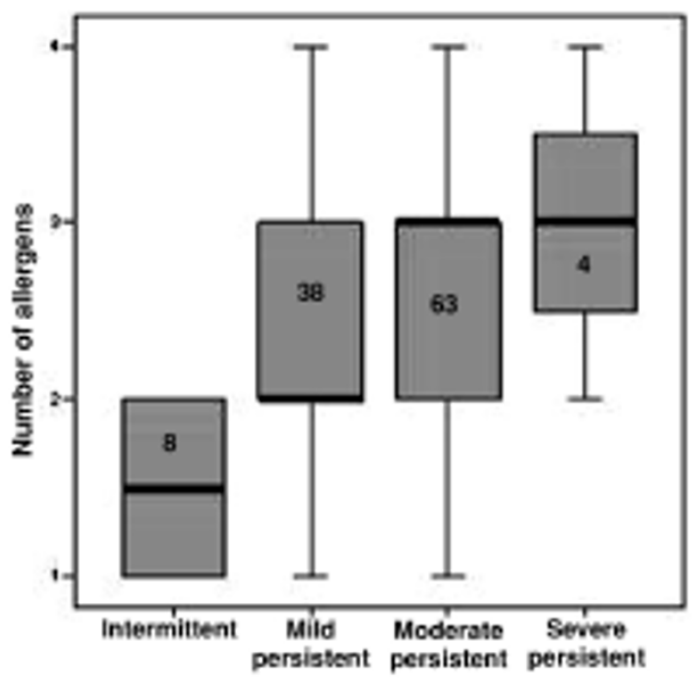

Figure 4 Number of allergens yielding sensitization, plotted against asthma severity. 
view of selection bias, the prevalence of sensitization in the general asthmatic population in Jeddah is most likely lower and needs investigation.

Sensitization to the two species of HDM was the predominant indoor allergen sensitization in more than $80 \%$ of the asthmatic patients. This pattern of sensitization is expected in a humid coastal city such as Jeddah. Conditions for mite growth are a temperature between $22^{\circ}$ and $26^{\circ} \mathrm{C}$ and a relative humidity $>55 \% . D p$ is the dominant mite in constantly damp climates; $D f$ survives better in somewhat drier climates. Modern houses are characterized by wall-to-wall carpeting, box mattresses, and optimal temperatures for the growth of HDMs. Worldwide, there is evidence to suggest that HDMs are the most common indoor allergens associated with asthma..$^{8,9}$

Alferah and colleagues conducted a study to analyze HDM content in samples collected from asthmatic patients' indoor environments in four regions of Saudi Arabia. ${ }^{15}$ The humid western region showed a high concentration of HDM (particularly more $\operatorname{Der} f \mathrm{I}$ ). Variations in both qualitative and quantitative assessments of HDMs may be attributed to variations in geography and climate, particularly humidity. SPT results with HDM allergens also revealed a high positivity rate, consistent with the concentration of HDMs in the region. ${ }^{15}$ This shows the possible influence of mites in patients' allergic manifestations, which are not only common but are also increasing in parts of the country. Airborne allergens were identified as risk factors for asthma and other allergic diseases in other Arabian Gulf countries. ${ }^{24}$

In this study, sensitization to cat allergen was evident in nearly half of the patients with positive skin test results, and sensitization to cockroach allergen was evident in one-third of them. Cat allergen is responsible for the rapid onset of respiratory symptoms in cat-sensitized persons entering an indoor environment that contains a cat and may constitute a relevant risk factor for asthma exacerbations. ${ }^{25,26}$ Additionally, public places and homes without a cat may contain sufficient allergenic protein to induce clinical symptoms in highly sensitized persons. ${ }^{27,28}$ In some locations, sensitization to cockroach allergen may be as common as sensitization to domestic mite allergens and can have a greater effect on asthma morbidity. ${ }^{29-31}$

Some indoor fungi (moulds) are an established risk factor for asthma in various populations. ${ }^{6,32}$ Future studies on the impact of other indoor allergens, such as moulds, on asthma in Saudi Arabia are needed.

This study demonstrated a correlation between the degree of IgE-mediated skin test reactivity to indoor allergens, particularly HDMs, and higher levels of clinical severity of asthma. The correlation found was low, and it was highly dependent on the four subjects in the severe persistent category. In other regions of the world, such a correlation has been recognized by several investigators. ${ }^{20,33,34}$ The strength of the IgE antibody response to HDMs in humid climates could contribute to the increased prevalence and severity of asthma. ${ }^{30,34}$

Recent asthma guidelines have established that great attention should be given to measures to prevent the symptoms of this chronic, lifelong, and incurable disease. ${ }^{35}$ Some measures for the prevention of asthma symptoms involve the avoidance of allergens and nonspecific triggers when asthma is established. Several studies have documented an improvement in asthma after exposure to the allergen ceases. ${ }^{36-38}$ Thus, indoor environmental control measures to reduce exposure to allergens might be important, but complete control is difficult to achieve, and there is conflicting evidence about whether such control measures are effective at reducing asthma symptoms..$^{39,40}$ Effective control strategies should be (1) tailored to individual allergens, (2) flexible for suiting individual needs, and (3) cost effective. ${ }^{35,41}$

HDMs are especially important in humid areas in Saudi Arabia, such as the cities of Jeddah and Dammam. ${ }^{15}$ According to asthma management guidelines, measures that significantly reduce exposure to mites must be an integral part of asthma management for asthmatic persons who have been sensitized to clinically relevant allergens. ${ }^{35,42}$ Environmental controls should be reinforced when asthma is poorly controlled or requires significant medication or whenever there is a suggestion that exposure to mites is playing a role..$^{35,42}$ The most effective and probably most important 
avoidance measure is to use mattress, pillow, and duvet covers that are impermeable to mite allergens (evidence level B). ${ }^{43-45}$

\section{Conclusion}

This work demonstrated that three-quarters of asthmatic persons living in Jeddah, Saudi Arabia, who were referred to the allergy clinic at King Abdulaziz University Hospital were sensitized to common indoor inhalant allergens. Additionally, there was a clear association between the degree of skin test reactivity to common indoor inhalant allergens and higher levels of clinical severity of bronchial asthma. Based on this, asthmatics should be offered effective education about the importance of exploring their sensitization to relevant environmental allergens. Subsequently, for better symptom control, health care workers must be encouraged to apply individualized educational strategies for the avoidance of allergens that are clinically relevant for their particular asthmatic patients. Eventually, this will be of significant help in the overall management of asthma symptoms.

\section{Acknowledgement}

This research was extrapolated from an unpublished study on the pattern of sensitization to common inhalant allergens in patients at King Abdulaziz University Hospital and was conducted during a year of sabbatical leave provided by King Abdulaziz University.

\section{References}

1. American Thoracic Society. Progress of the interface of inflammation and asthma. Am J Respir Crit Care Med 1995;152:385-9.

2. Robinson D. Allergen-induced airways inflammation in asthma. Monaldi Arch Chest Dis 1996;51:469-74.

3. Sporik R, Holgate ST, Platts-Mills TA, Cogswell JJ. Exposure to house-dust mite allergen (Der $p$ I) and the development of asthma in childhood.
A prospective study. N Engl J Med 1990; 323:502-7.

4. Platts-Mills TA. Role of allergens in asthma and airway hyperresponsiveness: relevance to immunotherapy and allergen avoidance. In: Kaliner MA, Persson CG, editors. Asthma: its pathology and treatment. New York: Marcel Dekker; 1991.

5. Newman-Taylor A. Environmental determinants of asthma. Lancet 1995;345:296-9.

6. O'Hollaren MT, Yunginger JW, Offord KP, et al. Exposure to an aeroallergen as a possible precipitating factor in respiratory arrest in young patients with asthma. N Engl J Med 1991; 324:359-63.

7. Djukanovic R, Feather I, Gratziou C, et al. Effect of natural allergen exposure during the grass pollen season on airways inflammatory cells and asthma symptoms. Thorax 1996;51:575-81.

8. Nelson HS. The importance of allergens in the development of asthma and the persistence of symptoms. J Allergy Clin Immunol 2000; 105:S628-32.

9. Platts-Mills TTA, Vervloet D, Thomas WR, et al. Indoor allergens and asthma: report of the Third International Workshop. J Allergy Clin Immunol 1997;100(6 Pt 1):S2-24.

10. Gold DR. Environmental tobacco smoke, indoor allergens, and childhood asthma. Environ Health Perspect 2000;108 Suppl 4:643-51.

11. Harrison PT. Creature comforts-living with mites and moulds. Clin Exp Allergy 1999; 29:148-9.

12. Hirsch T, Hering M, Burkner K, et al. Housedust-mite allergen concentrations (Der f 1 ) and mold spores in apartment bedrooms before and after installation of insulated windows and central heating systems. Allergy 2000;55:79-83.

13. Sporik R, Ingram JM, Price W, et al. Association of asthma with serum IgE and skin test reactivity to allergens among children living at high altitude. Tickling the dragon's breath. Am J Respir Crit Care Med 1995;151:1388-92.

14. Platts-Mills TA, Thomas WR, Aalberse RC, et al. Dust mite allergens and asthma: report of a second international workshop. J Allergy Clin Immunol 1992;89:1046-60.

15. Al-Frayh AS, Hasnain SM, Gad-El-Rab MO, et al. House dust mite allergens in Saudi Arabia: regional variations and immune response. Ann Saudi Med 1997;17:156-160. 
16. Burrows B, Martinez FD, Halonen M, et al. Association of asthma with serum IgE levels and skin test reactivity to allergens. $\mathrm{N}$ Engl J Med 1989;320:271-7.

17. Eriksson NE. Diagnosis of IgE mediated allergy in clinical practice. Allergol Immunopathol (Madr) 1994;22:139-51.

18. Gordon BR. Allergy skin tests for inhalants and foods. Comparison of methods in common use. Otolaryngol Clin North Am 1998;31:35-53.

19. Langley SJ, Goldthorpe S, Craven M, et al. Exposure and sensitization to indoor allergens: association with lung function, bronchial reactivity, and exhaled nitric oxide measures in asthma. J Allergy Clin Immunol 2003;112:362-8.

20. Sarpong SB, Karrison T. Skin test reactivity to indoor allergens as a marker of asthma severity in children with asthma. Ann Allergy Asthma Immunol 1998;80:303-8.

21. Al-Rayes H, Mobaireek K, Shimemreri A, Majeed S, editors. The national protocol for the management of asthma. 2nd ed. Saudi Arabia: Ministry of Health; 1997.

22. National Asthma Education Prevention Program (NAEPP). Expert Panel Report 2. National Institutes of Health (US); 1997 Mar. Publication No.: 97-3659.

23. Gerritsen J, Koeter GH, deMonchy JGR. Allergy in subjects with asthma from childhood to adulthood. J Allergy Clin Immunol 1990;85:116-25.

24. Bener A, Safa W, Abdulhalik S, Lestringant GG. An analysis of skin prick test reactions in asthmatics in a hot climate and desert environment. Allerg Immunol (Paris) 2002;34:281-6.

25. Custovic A, Simpson A, Woodcock A. Importance of indoor allergens in the induction of allergy and elicitation of allergic disease. Allergy 1998;53:115-20.

26. Liccardi G, Cazzola M, D'Amato M, D'Amato G. Pets and cockroaches: two increasing causes of respiratory allergy in indoor environments. Characteristics of airways sensitization and prevention strategies. Respir Med 2000;94:1109-18.

27. Partti-Pellinen K, Marttila O, Makinen-Kiljunen S, Haahtela T. Occurrence of dog, cat, and mite allergens in public transport vehicles. Allergy 2000;55:65-8.

28. Simpson A, Custovic A. Pets and the development of allergic sensitization. Curr Allergy Asthma Rep 2005;5:212-20.
29. Rosenstreich D, Eggleston P, Kattan M, et al. The role of cockroach allergy and exposure to cockroach allergen in causing morbidity among inner-city children with asthma. N Engl J Med 1997;336:1356-63.

30. Wood RA. House dust mite and cockroach exposure: risk factors for asthma. J Aerosol Med 2004;17:165-8.

31. Gruchalla R, Pongracic J, Plaut M, et al. Inner City Asthma Study: relationships among sensitivity, allergen exposure, and asthma morbidity. J Allergy Clin Immunol 2005; 115:478-85.

32. Halonen M, Stern DA, Wright AL, et al. Alternaria as a major allergen for asthma in children raised in a desert environment. Am J Respir Crit Care Med 1997;155:1356-61.

33. Kupczyk M, Kuprys I, Gorski P, Kuna P. Aspirin intolerance and allergy to house dust mites: important factors associated with development of severe asthma. Ann Allergy Asthma Immunol 2004;92:453-8.

34. Erwin EA, Wickens K, Custis NJ, et al. Cat and dust mite sensitivity and tolerance in relation to wheezing among children raised with high exposure to both allergens. J Allergy Clin Immunol 2005;115:74-9.

35. Global strategy for asthma management and prevention. Management segment (chapter 7). Washington (DC): National Institutes of Health (US); 1995 Jan. (2004 Update). NIH Publication No: 02-3659.

36. von Mutius E, Martinez FD, Fritzsch C, et al. Prevalence of asthma and atopy in two areas of West and East Germany. Am J Respir Crit Care Med 1994;149:358-64.

37. Leung R, Ho P. Asthma, allergy, and atopy in three South-east Asian populations. Thorax 1994;49:1205-10.

38. Becker AB. Primary prevention of allergy and asthma is possible. Clin Rev Allergy Immunol 2005;28:5-16.

39. Gotzsche PC, Hammarquist C, Burr M. House dust mite control measures in the management of asthma: meta-analysis. BMJ 1998;317: 1105-10.

40. Gotzsche PC, Johansen HK, Hammarquist C, Burr ML. House dust mite control measures for asthma (Cochrane Review). Cochrane Database Syst Rev 2001;2. 
41. Custovic A, Simpson A, Chapman MD, Woodcock A. Allergen avoidance in the treatment of asthma and atopic disorders. Thorax 1998;53:63-72.

42. Al- Frayh A, Khoja T, Al-Majed S, et al, editors. The national protocol for the management of asthma. 3rd edition. Saudi Arabia; Ministry of Health; 2000.

43. Ehnert B, Lau-Schadendorf S, Weber A, et al. Reducing domestic exposure to dust mite allergen reduces bronchial hyperreactivity in sensitive children with asthma. J Allergy Clin Immunol 1992;90:135-8.

44. Carswell F, Birmingham K, Oliver J, et al. The respiratory effects of reduction of mite allergen in the bedrooms of asthmatic children-a doubleblind controlled trial. Clin Exp Allergy 1996;26:386-96.

45. van der Heide S, Kauffman HF, Dubois AEJ, de Monchy JGR. Allergen reduction measures in houses of allergic asthmatic patients: effects of air-cleaners and allergen-impermeable mattress covers. Eur Respir J 1997;10:1217-23. 